\title{
Cost-Effectiveness of Strategies to Improve HIV Testing and Receipt of Results: Economic Analysis of a Randomized Controlled Trial
}

\author{
Gillian D. Sanders, $P h D^{7}$, Henry D. Anaya, PhD ${ }^{2,3}$, Steven Asch, MD, MPH', ${ }^{2,4}$, Tuyen Hoang, $P h D^{2}$, \\ Joya F. Golden, MSW2, Ahmed M. Bayoumi, MD, MSc ${ }^{5,6}$, and Douglas K. Owens, MD, MS ${ }^{7,8}$
}

'Duke Clinical Research Institute, Duke University, Durham, NC, USA; ${ }^{2}$ Veterans Affairs (VA) Greater Los Angeles Healthcare System, Los Angeles, CA, USA; ${ }^{3}$ UCLA School of Medicine, Los Angeles, CA, USA; ${ }^{4}$ RAND Health, Santa Monica, CA, USA; ${ }^{5}$ St. Michael's Hospital, Toronto, ON, Canada; ${ }^{6}$ University of Toronto, Toronto, ON, Canada; ${ }^{7}$ VA Palo Alto Healthcare System, Palo Alto, CA, USA; ${ }^{8}$ Stanford University, Stanford, CA, USA.

BACKGROUND: The CDC recommends routine voluntary HIV testing of all patients 13-64 years of age. Despite this recommendation, HIV testing rates are low even among those at identifiable risk, and many patients do not return to receive their results.

OBJECTIVE: To examine the costs and benefits of strategies to improve HIV testing and receipt of results. DESIGN: Cost-effectiveness analysis based on a Markov model. Acceptance of testing, return rates, and related costs were derived from a randomized trial of 251 patients; long-term costs and health outcomes were derived from the literature.

SETTING/TARGET POPULATION: Primary-care patients with unknown HIV status.

INTERVENTIONS: Comparison of three intervention models for HIV counseling and testing: Model A = traditional HIV counseling and testing; Model $\mathrm{B}=$ nurse-initiated routine screening with traditional HIV testing and counseling; Model $\mathrm{C}=$ nurse-initiated routine screening with rapid HIV testing and streamlined counseling.

MAIN MEASURES: Life-years, quality-adjusted life-years (QALYs), costs and incremental cost-effectiveness.

KEY RESULTS: Without consideration of the benefit from reduced HIV transmission, Model A resulted in perpatient lifetime discounted costs of $\$ 48,650$ and benefits of 16.271 QALYs. Model B increased lifetime costs by $\$ 53$ and benefits by 0.0013 QALYs (corresponding to 0.48 quality-adjusted life days). Model C cost \$66 more than Model A with an increase of 0.0018 QALYs (0.66 qualityadjusted life days) and an incremental cost-effectiveness of $\$ 36,390 /$ QALY. When we included the benefit from reduced HIV transmission, Model C cost \$10,660/QALY relative to Model A. The cost-effectiveness of Model C was robust in sensitivity analyses.

CONCLUSIONS: In a primary-care population, nurseinitiated routine screening with rapid HIV testing and

Electronic supplementary material The online version of this article (doi:10.1007/s11606-010-1265-5) contains supplementary material, which is available to authorized users.

Received July 27, 2009

Revised January 4, 2010

Accepted January 8, 2010

Published online March 4, 2010 streamlined counseling increased rates of testing and receipt of test results and was cost-effective compared with traditional HIV testing strategies.

KEY WORDS: HIV; cost-benefit analysis; highly active antiretroviral therapy; transmission; nurse-initiated HIV screening; HIV rapid testing; Streamlined counseling.

J Gen Intern Med 25(6):556-63

DOI: $10.1007 / \mathrm{s} 11606-010-1265-5$

(C) The Author(s) 2010. This article is published with open access at Springerlink.com

\section{INTRODUCTION}

Efforts to identify people early in the course of HIV infection in the United States have had limited success. Approximately half of patients are identified with HIV only when they have advanced disease. ${ }^{1,2}$ Recent CDC estimates indicate $38.3 \%$ of patients had received an AIDS diagnosis within a year of their HIV diagnosis; another 6.7\% received an AIDS diagnosis from 1 to 3 years after their HIV diagnosis. ${ }^{3}$ The Centers for Disease Control and Prevention (CDC) estimates that approximately 232,000 people who have HIV in the US are not aware they are infected. ${ }^{4}$ Approximately $21 \%$ of infected individuals across the US are unaware of their HIV status; ${ }^{5}$ the transmission rate from HIV-infected individuals unaware of their infection is up to 3.5 times higher that from individuals who are aware of their infection. ${ }^{6}$ The toll from this lack of awareness is high: over 14,000 new infections per year are caused by people who do not know they have HIV. ${ }^{7,8}$

Based on these findings, and on studies that indicate that risk-based screening fails to identify many people with HIV, the CDC now recommends that screening for HIV should be performed routinely for all patients between the ages 13-64 in health-care settings. ${ }^{1}$ A recent guidance statement from the American College of Physicians also recommends routine screening. ${ }^{9}$ Routine screening for HIV is cost-effective, even with prevalence as low as $0.05 \%$ to $0.1 \%,{ }^{10-12}$ which likely includes most health-care settings. Screening is also costeffective for patients up to the age of 75 , if it is performed with streamlined counseling. ${ }^{13}$

Despite the strong rationale for screening, there are many potential barriers to implementation of routine testing for HIV, and even patients at high-risk often are not tested..$^{714-16} \mathrm{We}$ report here the cost-effectiveness of three alternative strategies 
for implementing HIV testing that were evaluated previously in a randomized control trial. ${ }^{17}$ The three strategies are traditional HIV counseling and testing, nurse-initiated routine screening with traditional counseling and testing, and nurseinitiated routine screening with streamlined counseling and rapid HIV testing. The cost-effectiveness of these strategies is important because the randomized trial demonstrated that nurse-initiated routine screening with streamlined counseling and rapid HIV testing results in substantially higher testing rates, higher rates of receiving test results, and equivalent sexual risk behaviors and HIV knowledge post-intervention, when compared to traditional HIV counseling and testing. ${ }^{17}$

\section{METHODS}

We estimated the health and economic effects of strategies to increase HIV testing rates and receipt of results using a decision model. We followed the recommendations of the Panel on CostEffectiveness in Health and Medicine for performing and reporting a cost-effectiveness analysis. ${ }^{18}$ We used the perspective of a perfect insurer, ${ }^{19}$ which uses costs to the insurer and patient, and corresponds to what most studies term a societal perspective. Both costs and benefits were discounted at a 3\% annual rate, ${ }^{20}$ and patients were followed for their lifetime.
We adapted a Markov model ${ }^{21,22}$ developed to assess the costeffectiveness of voluntary HIV screening in health-care settings ${ }^{12,13}$ using Decision Maker software. Calibration and validation of the decision model and its outputs occurred as an iterative process throughout its development. HIV natural history and treatment effects were compared to published estimates, and our assumptions were modified as needed to calibrate to these data. ${ }^{11,23-25}$

The model tracked a cohort of patients over their lifetime. Patients were offered HIV testing and received their results through one of three methods: (1) traditional HIV testing and counseling (Model A), (2) nurse-initiated screening with traditional HIV testing and counseling (Model B), or (3) nurseinitiated screening with rapid HIV testing and streamlined counseling (Model C) (Fig. 1). For each strategy, we replicated the rates of HIV testing and receipt of results observed in the RCT. ${ }^{17}$ Each month, the model assessed the patients' HIV status, whether their HIV was identified, the clinical course of HIV disease, the costs and consequences of HIV transmission and of highly active antiretroviral therapy (HAART) for patients identified with HIV and eligible for treatment. Estimates for HIV natural history, treatment, transmission of HIV and quality-oflife were derived from high-quality published literature and expert clinical judgment. Our methods have been described previously $^{12,13}$ (Appendix available online)

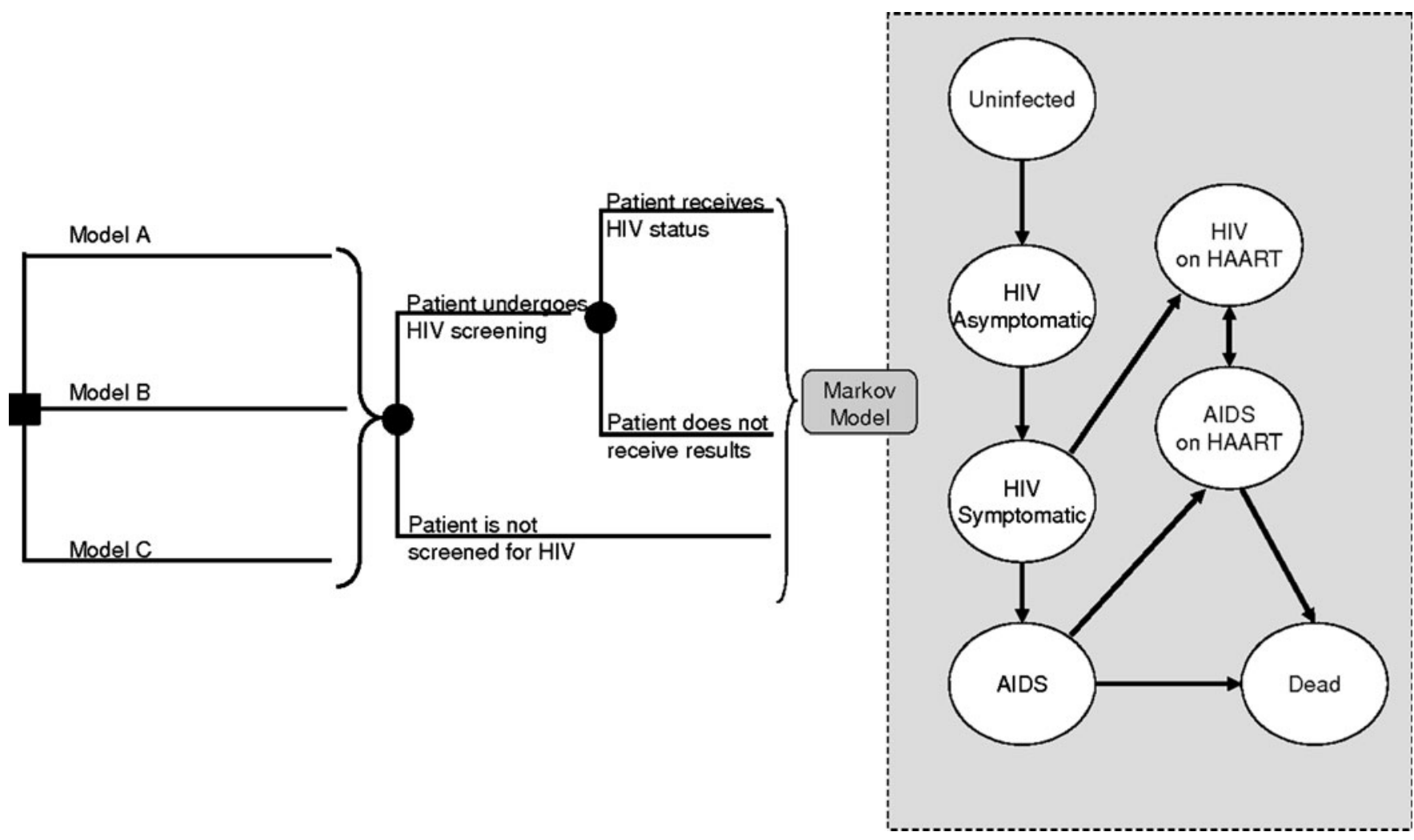

Figure 1. Schematic representation of decision model. The square node at the left represents the initial decision to initiate HIV testing through a physician-based or nurse-based strategy, and then whether traditional or streamlined counseling is performed. Patients in each strategy can then accept or refuse HIV screening. Once screened for HIV, patients could receive their test results or not. All patients regardless of their testing status then enter the Markov model (gray box). In all strategies, patients who do not receive screening through their initial interaction with the physician or nurse may be screened at a later date through symptom-based case finding. We assumed that the frequency with which case finding occurred was $80 \%$ annually below a CD4 count of 50 cells per cubic millimeter, linearly related to the CD4 count between 50 and 350 cells per cubic millimeter, and not relevant $(0 \%)$ with a CD4 count of more than 350 cells per cubic millimeter, when patients were assumed to be asymptomatic. Model $\mathrm{A}=$ traditional HIV counseling and testing; Model $\mathrm{B}=$ nurse-initiated screening with traditional HIV testing and counseling; Model $C=$ nurse-initiated screening with rapid HIV testing and streamlined counseling. 


\section{Patient Population}

Our cohort was modeled to reflect the patients in the RCT. ${ }^{17}$ The trial included 251 patients from two Department of Veterans Affairs (VA) sites in Southern California. The clinics were both primary-care clinics with urgent-care components. One was a large, university-affiliated hospital, and the other was an urban outpatient clinic serving many indigent and homeless veterans.

Patients were eligible for inclusion in the RCT if they met all of the following criteria: (1) aged 18-65 years, (2) unaware of their
HIV status, (3) had not received an HIV test in the past year, (4) had an appointment with a provider in the target clinic that day, (5) were proficient in English and (6) were competent to consent to the study. ${ }^{17}$

Patients in the trial were on average 49.7 years old, $32 \%$ White, 43\% African American, essentially all men, 9.6\% were men who have sex with men, and the prevalence of undiagnosed HIV in the population was $0.398 \%$ (Table 1$).{ }^{17}$ Age- and sexspecific incidence of future HIV in the patient population was based on previous models. ${ }^{4,8,26-28}$

Table 1. Input Variables and Sources

\begin{tabular}{|c|c|c|c|c|}
\hline Variable & Base case value & Range & Distribution $^{a}$ & Source \\
\hline \multicolumn{5}{|l|}{ Demographics } \\
\hline Age, years & 49.7 & $40-60$ & Normal & 17 \\
\hline Prevalence of undiagnosed HIV, \% & 0.398 & $0.1-5$ & Beta & 17 \\
\hline Men who have sex with men, $\%$ & 9.6 & $0-25$ & & 17 \\
\hline \multicolumn{5}{|l|}{ HIV test characteristics, $\%$} \\
\hline Sensitivity of traditional testing & 99.6 & $98-99.9$ & Beta & 12 \\
\hline Specificity of traditional testing & 99.9994 & $99-100$ & Beta & 12 \\
\hline Sensitivity of rapid testing & 99.6 & $98-100$ & Beta & http://www.fda.gov/cber/faq/oraqckfaq.htm \\
\hline Specificity of rapid testing & 99.9994 & $96-100$ & Discrete & Assumed to be equal to traditional testing, 47 \\
\hline \multicolumn{5}{|l|}{ Screening strategies } \\
\hline \multicolumn{5}{|l|}{ Probability of having an HIV test, \% } \\
\hline $\begin{array}{l}\text { Model A (traditional HIV counseling } \\
\text { and testing) }\end{array}$ & 41.0 & $30.2-51.8$ & Beta & 17 \\
\hline $\begin{array}{l}\text { Model B (nurse-initiated screening } \\
\text { with traditional counseling) }\end{array}$ & 84.5 & $76.7-92.3$ & Beta & 17 \\
\hline $\begin{array}{l}\text { Model C (nurse-initiated screening } \\
\text { with streamlined counseling and } \\
\text { rapid testing) }\end{array}$ & 89.3 & $82.5-96.1$ & Beta & 17 \\
\hline \multicolumn{5}{|l|}{$\begin{array}{l}\text { Probability of receiving HIV test result } \\
\text { (given test negative), \% }\end{array}$} \\
\hline Model A & 35.3 & $18.9-51.7$ & Beta & 17 \\
\hline Model B & 36.6 & $25.2-48.0$ & Beta & 17 \\
\hline Model C & 89.3 & $82.1-96.5$ & Beta & 17 \\
\hline \multicolumn{5}{|l|}{$\begin{array}{l}\text { Probability of receiving HIV test result } \\
\text { (given test positive), } \%\end{array}$} \\
\hline Model A & 90 & $30-100$ & Beta & Estimate and range based on $7,15,48$ \\
\hline Model B & 90 & $30-100$ & Beta & Estimate and range based on $7,15,48$ \\
\hline Model C & 100 & $80-100$ & Beta & Estimate and range based on $7,15,48$ \\
\hline \multicolumn{5}{|l|}{ Costs, $\$$} \\
\hline \multicolumn{5}{|l|}{ HIV screening, negative test, $\$$} \\
\hline Conventional screening & 12.41 & $9.30-15.50$ & & $\begin{array}{l}\text { CMS reimbursement rates for the VA for } \\
\text { CPT } 86701 \text { HIV-1 EIA }\end{array}$ \\
\hline Rapid test screening & 11.45 & $8.50-14.50$ & & Abbott Laboratories \\
\hline \multicolumn{5}{|l|}{ HIV screening, positive test, \$ } \\
\hline Conventional screening & 51.87 & $38.90-64.84$ & & $\begin{array}{l}\text { CMS reimbursement rates for the VA for } \\
\text { CPT } 86701 \text { HIV-1 EIA and second EIA } \\
\text { and CPT } 86689 \text { HIV-1 Western blot }\end{array}$ \\
\hline Rapid test screening & 50.91 & $38.18-63.64$ & & $\begin{array}{l}\text { CMS reimbursement rates for the VA for CPT } \\
86701 \text { HIV-1 EIA and second EIA and CPT } \\
86689 \text { HIV-1 Western blot incorporating } \\
\text { rapid test screening cost }\end{array}$ \\
\hline \multicolumn{5}{|l|}{ Pre-test counseling, \$ } \\
\hline Conventional counseling & 10.16 & $6.77-13.55$ & Gamma & $\begin{array}{l}\text { Based on } 15 \mathrm{~min} \text { (range } 10-20 \mathrm{~min} \text { ) for an HIV } \\
\text { counselor at a average salary of } \$ 81,307\end{array}$ \\
\hline Streamlined counseling & 5.00 & $0.30-9.69$ & Gamma & $\begin{array}{l}\text { Based on } 7.38 \mathrm{~min} \text { (standard deviation } \\
6.9 \mathrm{~min} \text { ) for an HIV counselor at a average } \\
\text { salary of } \$ 81,307\end{array}$ \\
\hline $\begin{array}{l}\text { Post-test counseling for negative } \\
\text { results, \$ }\end{array}$ & 1.36 & $0.68-6.78$ & & $\begin{array}{l}\text { Based on } 2 \text { min (range } 1-10 \mathrm{~min} \text { ) for an } \\
\text { HIV counselor at an average salary of } \$ 81,307 \text {. } \\
\text { Same cost for post-test counseling of negative } \\
\text { results regardless of testing type }\end{array}$ \\
\hline $\begin{array}{l}\text { Post-test counseling for positive } \\
\text { results, } \$\end{array}$ & 13.55 & $20.33-40.65$ & & $\begin{array}{l}\text { Based on } 20 \mathrm{~min} \text { (range } 10-60 \mathrm{~min} \text { ) for an HIV } \\
\text { counselor at an average salary of } \\
\$ 81,307 \text {. Same cost for post-test counseling } \\
\text { of positive results regardless of testing type }\end{array}$ \\
\hline
\end{tabular}

${ }^{a}$ Indicates the distribution used in the probabilistic sensitivity analysis 


\section{HIV Testing and Receipt of Result Strategies}

The strategies in our analysis reflected those of the RCT. In strategy Model A, patients were encouraged by a nurse to discuss their need for a HIV-screening test with their physician. If the patient and physician agreed on HIV screening, the test, as well as the pre- and post-test counseling, was based on standard pretest and posttest counseling performed by a trained counselor. HIV screening was performed using a HIV1 serum enzyme-linked immunosorbent assay (EIA) test and, if positive, was followed by a repeat HIV-1 EIA test and a HIV-1 Western blot analysis. Per the published literature, we assumed traditional HIV testing had a sensitivity of $99.6 \%$ and a specificity of $99.9994 \% .^{12}$

In the Model B strategy, the nurse recommended that the patient get screened for HIV and, if the patient agreed, they were then referred for traditional counseling/testing.

Model C involved the nurse recommending HIV screening to the patient. If the patient agreed to testing, then the patient was screened using an oral swab and the OraQuick ${ }^{\circledR}$ rapid screening test (OraSure Technologies, Inc, Bethlehem PA). Sensitivity of rapid HIV testing was based on OraQuick data and assumed to be $99.6 \%$. The pre-test counseling was performed using a streamlined procedure ${ }^{1}$ that took approximately $7 \mathrm{~min}$. Post-test counseling and follow-up of positive rapid HIV tests were performed in all strategies by a trained HIV counselor.

The trial indicated that traditional HIV counseling and testing (Model A) resulted in lower rates of testing (41\%) and receipt of results given testing (35.3\%) than did the nurse-initiated strategies (Models B and C), as shown in Table 1. The most successful strategy was Model C, nurse-initiated routine screening with streamlined counseling and rapid HIV testing, in which approximately $90 \%$ of patients were tested and $90 \%$ of those tested received results (Table 1).

\section{Quality of Life}

The model incorporated adjustments for the quality of life associated with age-specific current health, HIV disease (asymptomatic HIV, symptomatic HIV and AIDS) and HAART. ${ }^{12}$ Utilities measure the patient's quality of life and were rated on a scale from 0 to 1 , where 0 represents death and 1 ideal health. We multiplied utilities based on HIV-related health status and knowledge by age-specific utility weights derived from the Beaver Dam Health Outcomes Study. ${ }^{29}$

\section{Costs}

We included the direct costs of medical care associated with physician and nurse time, HIV testing (initial and confirmatory tests) and counseling, follow-up and treatment of patients identified with HIV infection (Table 1). We did not include costs associated with patient time. Costs of conventional HIV testing were derived from Centers for Medicare and Medicaid (CMS) reimbursement rates for the VA, while cost of rapid HIV testing was estimated from Abbott Laboratories. Costs of conventional pre-test and post-test counseling were based on the estimated time needed for a VA-based HIV counselor and using an average annual salary of $\$ 81,300$ (Table 1 ). The time required for streamlined counseling prior to HIV testing was determined through a time-costing analysis in the RCT (average time $=7.4$ min). Costs of HIV treatment and follow-up were based on our earlier analysis. ${ }^{12,13}$ We included age-specific medical expenditures unrelated to HIV care based on data from the Bureau of Labor Statistics (http://www.bls.gov/cex/csxann05.pdf). All costs were updated to 2007 dollars. ${ }^{30}$

\section{Sensitivity Analyses}

We performed one-way and multi-way sensitivity analyses to account for important model assumptions and uncertainties. For variables derived from the randomized trial, ranges represent the 95\% confidence intervals reported in the trial. For HIV natural history and clinical variables, our ranges for sensitivity analyses represent our judgment of the variation likely to be encountered in clinical practice on the basis of both the literature and discussion with experts. We varied costs in sensitivity analyses based on the standard deviation observed in the time-costing analysis, expert clinical judgment or assuming costs increased/decreased by $25 \%$. A probabilistic sensitivity analysis was also performed, and its assumptions and findings are described in the Appendix available online.

\section{RESULTS}

We estimated the increase in life expectancy and qualityadjusted life expectancy that resulted from identification and treatment of HIV through the nurse-initiated screening (Models B and C) compared with traditional HIV counseling and testing. For an HIV-infected patient (49.7 years), Model B increased life expectancy by 0.64 years or 0.47 QALYs compared with Model A. If the nurse-initiated strategy included rapid testing and streamlined counseling (Model C), life expectancy was increased by 0.87 years or 0.63 QALYs compared to Model A (Fig. 2).

Model A resulted in the lowest costs and effectiveness (Table 2 and Fig. 3). Model B was more effective and costly, and Model $\mathrm{C}$ was the most effective and most costly (Table 2). Note that lifetime costs in Table 2 include all direct medical costs for screening, counseling and treatment, as well as age-specific medical costs unrelated to HIV care, and that our estimated HIV prevalence of $0.398 \%$ was based on the trial results. Although Model B is more effective than Model A, Model C has a more favorable cost-effectiveness ratio than does Model B; thus Model $\mathrm{B}$ is eliminated by extended dominance (Table 2). Extended dominance occurs when a more expensive strategy has a lower cost-effectiveness ratio than a competing (dominated) strategy; in this case, the more expensive alternative would always be preferable, since the outcome gain more than compensates for the cost. The additional gains in health benefit from Model $\mathrm{C}$ cost $\$ 36,390 /$ BALY, without consideration of the benefit from reduced transmission. Including these benefits resulted in a more favorable cost-effectiveness ratio of \$10,660/QALY (Table 2, Fig. 3).

\section{Sensitivity Analyses}

If the prevalence of unidentified HIV was lowered from $0.398 \%$ to $0.05 \%$, the cost-effectiveness of Model C compared with Model A was less favorable at $\$ 58,900 /$ QALY (Fig. 4) without the benefits from reduced transmission; the cost-effectiveness was $\$ 25,300 /$ GALY with the benefit from reduced transmission included. Including the benefits to partners, Model $\mathrm{C}$ cost less than $\$ 75,000 /$ QALY as long as the prevalence of unidentified HIV was greater than $0.01 \%$. 


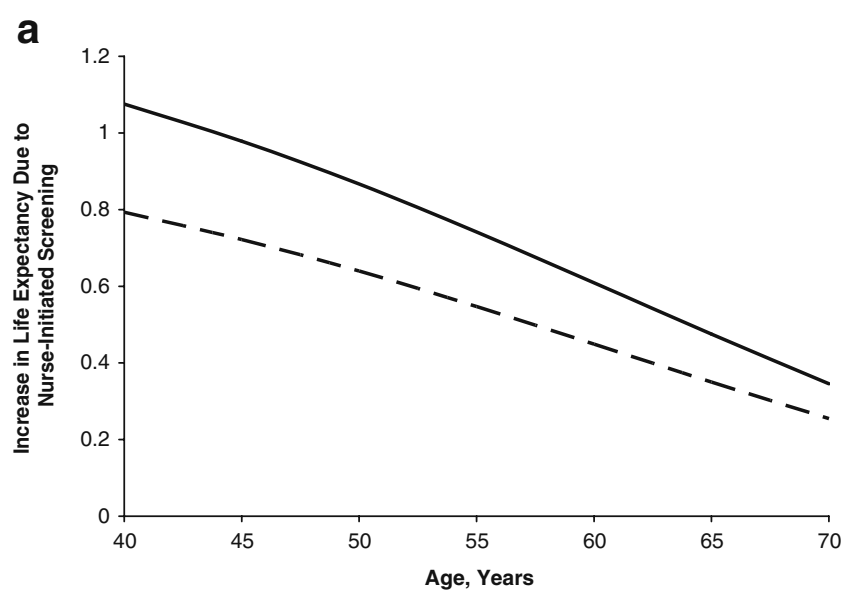

b

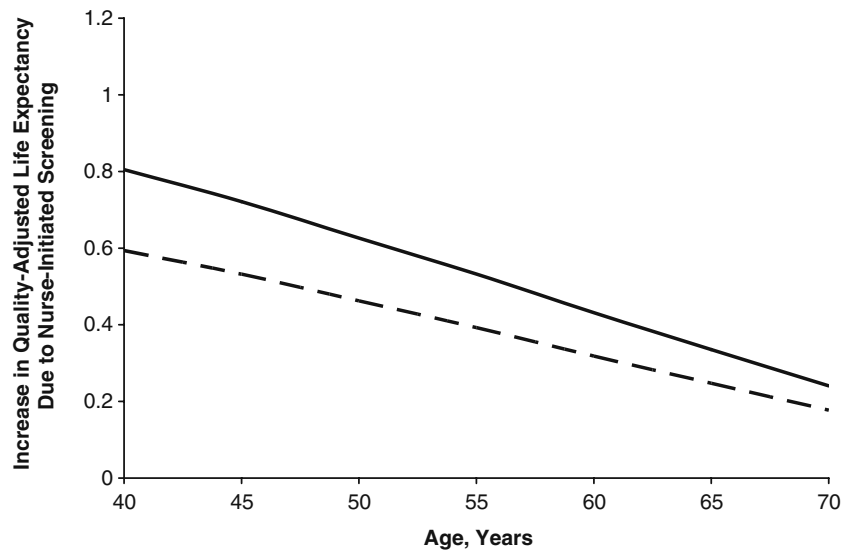

Figure 2. Effect of nurse-initiated screening strategies on life expectancy of HIV-infected patients. The effect on (a) undiscounted life expectancy and (b) undiscounted quality-adjusted life expectancy of using nurse-initiated testing for HIV infection, as compared with traditional counseling and testing (Model A). The solid line demonstrates the benefit to HIV-infected patients from incorporating streamlined counseling and rapid testing into the nurse-initiated strategy (Model C), while the dashed line represents nurse-initiated testing with traditional counseling (Model B).
The benefits of Model $\mathrm{C}$ to patients are two-fold (i.e., the probability of a patient receiving an HIV test is increased, and given that HIV testing occurs, patients were more likely to receive these results). In sensitivity analyses we explored the effect of these two elements. If $89.3 \%$ of patients received their test results through rapid-testing and streamlined counseling (base-case estimates), then even if the HIV test acceptance rate was reduced to $40 \%$, the cost-effectiveness ratio of Model C increased from the base case estimate of $\$ 10,660 /$ QALY only to $\$ 13,240 /$ QALY.

Sensitivity analyses with other model variables (Table 1) did not change our results substantially. If the specificity of rapid testing was reduced to $96.1 \%$ and all positive tests were confirmed by an EIA, Western blot and viral load tests, Model C remained cost-effective at $\$ 17,400 /$ QALY.

\section{DISCUSSION}

We evaluated the cost-effectiveness of three strategies for implementing routine HIV screening. We used testing and counseling costs and receipt of test result outcomes from a previously performed randomized trial as inputs to a modelbased analysis that estimated lifetime costs and health outcomes. This approach enabled us to assess the cost-effectiveness of these strategies by including both short- and long-term costs and health outcomes, in accordance with guidelines for the conduct of cost-effectiveness analyses. ${ }^{18}$

Our main finding is that nurse-initiated routine screening with streamlined counseling and rapid HIV testing is cost-effective relative to traditional HIV counseling and testing. The increased effectiveness and the resulting cost-effectiveness occur because this strategy resulted in much higher rates of receipt of test results. This strategy, at $\$ 36,390 /$ QALY gained, met conventional thresholds for cost-effectiveness relative to Model A. When we considered the benefit to sexual partners from reduced transmission, rapid testing with streamlined counseling was even more favorable and cost $\$ 10,660 /$ QALY gained.

Although the CDC now recommends routine HIV screening in all health-care settings, implementation of screening remains a

Table 2. Health and Economic Outcomes

\begin{tabular}{|c|c|c|c|c|c|c|}
\hline \multirow[t]{2}{*}{ Outcome } & \multicolumn{3}{|c|}{ Benefits to partners excluded } & \multicolumn{3}{|c|}{ Benefits to partners included } \\
\hline & Model A & Model B & Model C & Model A & Model B & Model C \\
\hline Patients tested, \% & 41.0 & 84.5 & 89.3 & 41.0 & 84.5 & 89.3 \\
\hline Tested patients who receive results, \% & 35.3 & 36.6 & 89.3 & 35.3 & 36.6 & 89.3 \\
\hline Lifetime cost, \$ & 48,650 & 48,710 & 48,720 & 49,040 & 49,060 & 49,070 \\
\hline Incr. cost, \$ & & 53 & 13 & & 27 & 4 \\
\hline LY, years & 18.8330 & 18.8348 & 18.8355 & 18.8153 & 18.8178 & 18.8187 \\
\hline Incr. LY, years (life days) $^{\mathrm{a}}$ & & $0.0018(0.65)$ & $0.0007(0.25)$ & & $0.0025(0.91)$ & $0.0009(0.34)$ \\
\hline Incremental cost-effectiveness, \$/LY & & Extended dominance $^{\mathrm{b}}$ & $26,710^{\mathrm{c}}$ & & Extended dominance $^{\mathrm{b}}$ & $9,240^{\mathrm{c}}$ \\
\hline QALY, years & 16.2714 & 16.2727 & 16.2732 & 16.2530 & 16.2551 & 16.2559 \\
\hline Incr. QALY, years (days) $^{\mathrm{a}}$ & & $0.0013(0.48)$ & $0.0005(0.19)$ & & $0.0021(0.77)$ & $0.0008(0.29)$ \\
\hline Incremental cost-effectiveness, \$/QALY & & Extended dominance $^{\mathrm{b}}$ & $36,390^{c}$ & & Extended dominance $^{\mathrm{b}}$ & $10,660^{\mathrm{c}}$ \\
\hline
\end{tabular}

${ }^{a}$ One life year $(L Y)=365$ life days; one quality-adjusted life year $(B A L Y)=365$ quality-adjusted life days

${ }^{b}$ Extended dominance = extended dominance occurs when a more expensive strategy has a more favorable cost-effectiveness ratio than a less expensive strategy; in this case, the more expensive alternative would always be preferable, since the outcome gain more than compensates for the cost

${ }^{c}$ Because the Model B strategy is eliminated through extended dominance, the incremental cost-effectiveness ratios listed compare the Model $C$ strategy directly to the Model A strategy. Model $A=$ traditional counseling and testing; Model $B=$ nurse-initiated screening with traditional counseling, Model $C=$ nurse-initiated screening with streamlined counseling and rapid testing 


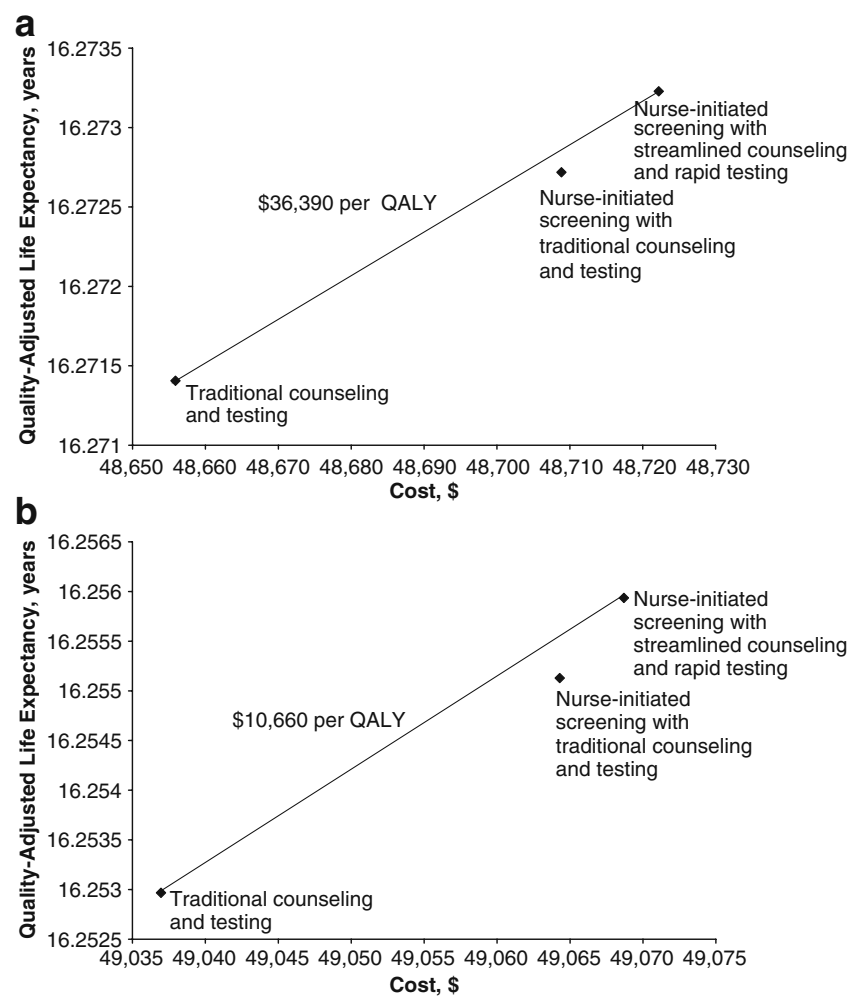

Figure 3. Health and economic outcomes of testing and counseling strategies. (a) Benefits to partners excluded; (b) benefits to partners included.

challenge, and few health-care systems have integrated screening into routine care. We believe that three elements of Model C contributed to the success of this strategy. First, the use of nonphysician providers, in our case, nurses, substantially increased the rate of testing. This finding is consistent with a substantial body of literature that supports the use of non-physician providers for preventive care. ${ }^{31-36}$ For example, a systematic review of 81 studies found that the most potent type of intervention to increase preventive services was organizational change, including designation of non-physician staff to perform specific prevention activities. ${ }^{36}$ Use of non-physician providers has the additional potential advantage of reducing per-person test costs by substitution of less expensive personnel. In our trial, aggregate costs were higher for the strategies that used nurse-initiated screening (Models B and C), but the increase in costs was related to the higher rates of testing, which offset the reduction in time and personnel costs.

The second important component of this strategy was streamlined counseling. With traditional testing and counseling, counseling costs are substantial, and for patients with negative tests are approximately 15 times higher than the cost of the HIV test itself. ${ }^{12}$ In the randomized trial, traditional pre-test counseling required $20 \mathrm{~min}$ on average. In contrast, streamlined pretest counseling took $7 \mathrm{~min}$, which resulted in a reduction in counseling costs of $65 \%$. In the trial, there were no differences in HIV knowledge or in sexual risk behavior between the traditional or streamlined counseling groups. ${ }^{17}$ This result is consistent with that of the RESPECT-2 randomized trial of over 1,600 patients that compared rapid HIV testing and counseling in one visit to standard testing and counseling over two visits. ${ }^{37}$ However, the counseling for rapid testing in RESPECT-2 required $30 \mathrm{~min}$ and was likely both more costly and more intensive than our streamlined counseling. In addition, in posthoc subgroup analyses in RESPECT-2, one time counseling appeared modestly less effective in men, suggesting that further evaluation of streamlined counseling is needed.

The third important component of this strategy was rapid testing. Rapid testing enabled patients to receive test results in approximately $20 \mathrm{~min}$, whereas traditional testing required two visits and results were not available for 1 to 2 weeks. In our trial, the test costs were similar for rapid versus traditional testing. Our results about receipt of testing are consistent with a number of studies that indicate that rapid testing substantially increases the receipt of results ${ }^{16,38-41}$ and is acceptable to patients. ${ }^{42,43}$ Recent studies have also indicated the potential economic benefits of rapid testing strategies. ${ }^{44,45}$

Although the convenience of rapid testing is an important advantage, continued evaluation of the accuracy of rapid testing is necessary. In our trial, we had only one positive result, which was a true positive; thus, the false-positive rate was 0 . However, a trial of testing in emergency department with rigorous quality control found a false-positive rate of approximately $3.1 \%{ }^{46}$ Patients should be counseled that positive rapid tests require confirmation.

Our trial was performed at two VA primary-care clinics in southern California, which raises the question of whether the results will be applicable in other settings. Although the rates of acceptance and receipt of result are likely to vary across settings, we believe the three components of our most effective strategy (initiation of testing by non-physician providers, use of streamlined counseling and rapid testing) are likely to be useful in other settings. Rapid testing has been evaluated in many environments, and use of non-physician providers has been studied in many prevention domains, as noted previously. The use of streamlined counseling addresses an important challenge to providers who have many competing demands on their time.

Our study has several limitations. As noted, our trial was performed in VA primary and urgent care settings, which have different patient populations than many primary or urgent care practices. In our trial, about $17 \%$ of patients approached for participation agreed to enter the study. Because this was a

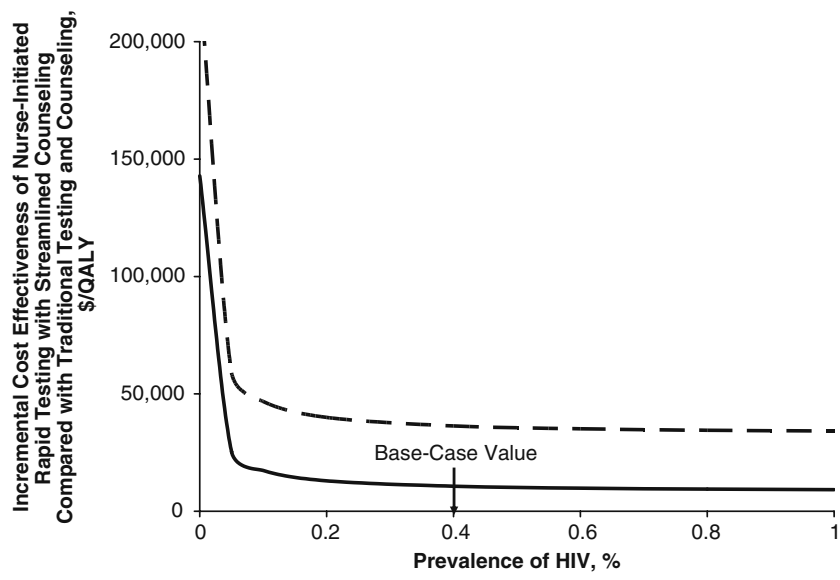

Figure 4. Sensitivity analysis of the effect of unidentified HIV prevalence on the incremental cost-effectiveness of the nurseinitiated rapid testing and streamlined counseling strategy (Model C) compared with traditional counseling and testing (Model A). The solid line includes the costs and benefits to partners, while the dashed line excludes these effects. 
research study, informed consent was required, and the requirements for follow-up may have discouraged some patients from participating. Thus, the implications for implementation of screening outside a trial are not known. The cost-effectiveness of screening however would not be affected by the participation rate since a change in participation would increase/decrease costs and benefits proportionally. In addition, the VA populations we studied do not reflect the distributions or the risk groups in some other populations or settings. Because our results may not be generalizable to non-VA setting, further study in other settings would be helpful. In addition, longer term assessment of effectiveness of streamlined counseling would be useful; our follow-up did not extend beyond 4 weeks. Finally, our costeffectiveness analysis assumed that identified patients would have access to HIV care, which is true in the VA, but may not hold in some settings. The benefit from screening would be less than we estimated if patients did not have full access to care.

We believe our study is relevant to primary care settings. Although the need for informed consent in our study precludes completely reproducing routine opt-out screening in a medical setting, we did offer testing routinely to all patients, which is in essence the $\mathrm{CDC}$ recommendation. We note, however, that there are potential barriers to implementation of nurse-based screening with rapid testing (Model C), which include lack of nursing time, prioritization of other tasks over HIV testing, lack of familiarity with rapid test procedures among nursing staff and the logistics of lengthening clinic encounters because patients must wait for test results. The importance of these barriers is likely to vary by clinical setting.

The current CDC guidelines recommend routine screening in health settings, a substantial change in policy from targeted riskbased screening. How best to implement routine screening is uncertain. We found that nurse-initiated screening with streamlined counseling and rapid testing (Model C) was an effective strategy to increase testing and receipt of results, and is costeffective by conventionally accepted thresholds when compared with traditional counseling/testing. Our study suggests that this strategy has substantial promise as an approach for integrating routine HIV screening into the practice of primary care.

Acknowledgments: This project was supported by the Department of Veterans Affairs Health Services Research and Development Service and the National Institute on Drug Abuse (R01 DA15612-01). Dr. Bayoumi was supported by a career scientist award from the Ontario HIV Treatment Network. The authors gratefully acknowledge the support of the Ontario Ministry of Health and Long-Term Care. The views expressed in this article are those of the author(s) and do not necessarily represent the views of the United States Department of Veterans Affairs or of the Ontario Ministry of Health and Long-Term Care. The funders had no role in the design or implementation of the study or in the decision to seek publication.

Conflict of Interest: HIV rapid tests for the randomized trial were donated by Orasure Technologies. Dr. Anaya owns stock in a biotechnology company that develops biotechnological products, one of which is a rapid test for diagnosing the HIV virus. Drs. Anaya and Asch have also received an unrestricted grant to support dissemination of research results from two HIV rapid testing device manufacturers. All remaining authors do not have any industry support or potential conflicts of interest.

This study was approved by the Institutional Review Board at each participating institution.

The authors made the following contributions to the manuscript:

Gillian D. Sanders PhD: conception and design, acquisition of data, analysis and interpretation of data, drafting of the manu- script, critical revision of the manuscript for important intellectual content, statistical analysis, supervision

Henry D. Anaya PhD: conception and design, acquisition of data, analysis and interpretation of data, critical revision of the manuscript for important intellectual content, obtaining funding

Steven Asch MD, MPH: conception and design, acquisition of data, analysis and interpretation of data, critical revision of the manuscript for important intellectual content, obtaining funding, supervision

Tuyen Hoang PhD: acquisition of data, analysis and interpretation of data, critical revision of the manuscript for important intellectual content, statistical analysis

Joya F Golden MSW: acquisition of data, critical revision of the manuscript for important intellectual content, statistical analysis

Ahmed M. Bayoumi MD, MSc: analysis and interpretation of data, critical revision of the manuscript for important intellectual content, statistical analysis

Douglas K. Owens MD, MS: conception and design, acquisition of data, analysis and interpretation of data, drafting of the manuscript, critical revision of the manuscript for important intellectual content, obtaining funding, supervision

Open Access: This article is distributed under the terms of the Creative Commons Attribution Noncommercial License which permits any noncommercial use, distribution, and reproduction in any medium, provided the original author(s) and source are credited.

Corresponding Author: Gillian D. Sanders, PhD; Duke Clinical Research Institute, Duke University, PO Box 17969, Durham, NC 27715, USA (e-mail: gillian.sanders@duke.edu).

\section{REFERENCES}

1. Branson BM, Handsfield HH, Lampe MA, et al. Revised recommendations for HIV testing of adults, adolescents, and pregnant women in health-care settings. MMWR Recomm Rep. 2006;55(RR-14):1-7. quiz CE1-4.

2. Wortley PM, Chu SY, Diaz T, et al. HIV testing patterns: where, why, and when were persons with AIDS tested for HIV? Aids. 1995;9(5): 48792.

3. Late HIV testing-34 states, 1996-2005. MMWR Morb Mortal Wkly Rep 2009;58(24):661-5.

4. HIV prevalence estimates-United States, 2006. MMWR Morb Mortal Wkly Rep. 2008;57(39):1073-6.

5. Campsmith ML, Rhodes PH, Hall HI, Green TA. Undiagnosed HIV prevalence among adults and adolescents in the United States at the end of 2006. J Acquir Immune Defic Syndr. 2009.

6. Marks G, Crepaz N, Janssen RS. Estimating sexual transmission of HIV from persons aware and unaware that they are infected with the virus in the USA. Aids. 2006;20(10):1447-50.

7. Centers for Disease Control and Prevention. Advancing HIV prevention: new strategies for a changing epidemic-United States, 2003. MMWR. 2003;52(15):329-32.

8. Hall HI, Song R, Rhodes $\mathbf{P}$, et al. Estimation of HIV incidence in the United States. Jama. 2008;300(5):520-9.

9. Gaseem A, Snow V, Shekelle P, Hopkins R Jr, Owens DK. Screening for HIV in health care settings: a guidance statement from the American College of Physicians and HIV Medicine Association. Ann Intern Med. 2009; 150(2): 125-31.

10. Paltiel AD, Walensky RP, Schackman BR, et al. Expanded HIV screening in the United States: effect on clinical outcomes, HIV transmission, and costs. Ann Intern Med. 2006;145(11):797-806.

11. Paltiel AD, Weinstein MC, Kimmel AD, et al. Expanded screening for HIV in the United States-an analysis of cost-effectiveness. N Engl J Med. 2005;352(6):586-95.

12. Sanders GD, Bayoumi AM, Sundaram V, et al. Cost-effectiveness of screening for HIV in the era of highly active antiretroviral therapy. N Engl J Med. 2005;352(6):570-85.

13. Sanders GD, Bayoumi AM, Holodniy M, Owens DK. Cost-effectiveness of HIV screening in patients older than 55 years of age. Ann Intern Med. 2008; 148(12):889-903.

14. Owens DK, Sundaram V, Lazzeroni LC, et al. HIV testing of at risk patients in a large integrated health care system. J Gen Intern Med. 2007;22(3):315-20. 
15. Tao G, Branson BM, Kassler WJ, Cohen RA. Rates of receiving HIV test results: data from the U.S. National Health Interview Survey for 1994 and 1995. J Acquir Immune Defic Syndr. 1999;22(4):395-400.

16. Hutchinson AB, Branson BM, Kim A, Farnham PG. A meta-analysis of the effectiveness of alternative HIV counseling and testing methods to increase knowledge of HIV status. Aids. 2006;20(12):1597-604.

17. Anaya HD, Hoang T, Golden JF, et al. Improving HIV screening and receipt of results by nurse-initiated streamlined counseling and rapid testing. J Gen Intern Med. 2008;23(6):800-7.

18. Gold MR, Seigel JE, Russell LB, Weinstein MC (eds). Cost-effectiveness in health and medicine. New York: Oxford University Press; 1996.

19. Garber AM. Recent developments in CBA/CEA. In: Culver AJ, Newhouse JP (eds) Handbook of health economics. 1 ed: Elsevier Science B.V; 2000.

20. Weinstein MC, Siegel JE, Gold MR, Kamlet MS, Russell LB. Recommendations of the Panel on Cost-effectiveness in Health and Medicine. Jama. 1996;276(15):1253-8.

21. Beck JR, Pauker SG. The Markov process in medical prognosis. Med Decis Making. 1983;3:419-58.

22. Sonnenberg FA, Beck JR. Markov models in medical decision making: a practical guide. Med Decis Making. 1993;13:322-38.

23. Koblin BA, van Benthem BH, Buchbinder SP, et al. Long-term survival after infection with human immunodeficiency virus type 1 (HIV-1) among homosexual men in hepatitis B vaccine trial cohorts in Amsterdam, New York City, and San Francisco, 1978-1995. Am J Epidemiol. 1999;150(10):1026-30.

24. Phillips AN, Leen C, Wilson A, et al. Risk of extensive virological failure to the three original antiretroviral drug classes over long-term follow-up from the start of therapy in patients with HIV infection: an observational cohort study. Lancet. 2007;370(9603):1923-8.

25. King JT Jr, Justice AC, Roberts MS, Chang CC, Fusco JS. Long-term HIV/AIDS survival estimation in the highly active antiretroviral therapy era. Med Decis Making. 2003;23(1):9-20.

26. Rosenberg PS. Scope of the AIDS epidemic in the United States. [see comments]. Science. 1995;270(5240):1372-5.

27. Karon JM, Fleming PL, Steketee RW, De Cock KM. HIV in the United States at the turn of the century: an epidemic in transition. Am J Public Health. 2001;91(7):1060-8.

28. Karon JM, Song R, Brookmeyer R, Kaplan EH, Hall HI. Estimating HIV incidence in the United States from HIV/AIDS surveillance data and biomarker HIV test results. Stat Med. 2008;27(23):4617-33.

29. Fryback DG, Dasbach EJ, Klein R, et al. The Beaver Dam Health Outcomes Study: initial catalog of health-state quality factors. Med Decis Making. 1993;13(2):89-102.

30. Executive Office of the President of the United States. Budget of the United States Government, Fiscal Year 2009: Historical tables in spreadsheet format. Section 10: 10.1- Gross Domestic Product and Deflators Used in the Historical Tables: 1940-2013 Gross Domestic Product and Implicit Outlay Deflators. Vol. 2008; 2008.

31. Atri J, Falshaw M, Gregg R, Robson J, Omar RZ, Dixon S. Improving uptake of breast screening in multiethnic populations: a randomised controlled trial using practice reception staff to contact non-attenders. Bmj. 1997;315(7119):1356-9.

32. Cargill VA, Conti M, Neuhauser D, McClish D. Improving the effectiveness of screening for colorectal cancer by involving nurse clinicians. Med Care. 1991;29(1):1-5.
33. Champion VL. Strategies to increase mammography utilization. Med Care. 1994;32(2):118-29.

34. Gonzalez JJ, Ranney J, West J. Nurse-initiated health promotion prompting system in an internal medicine residents' clinic. South Med J. 1989;82(3):342-4.

35. Robson J, Boomla K, Fitzpatrick S, et al. Using nurses for preventive activities with computer assisted follow-up: a randomised controlled trial. Bmj. 1989;298(6671):433-6.

36. Stone EG, Morton SC, Hulscher ME, et al. Interventions that increase use of adult immunization and cancer screening services: a metaanalysis. Ann Intern Med. 2002;136(9):641-51.

37. Metcalf CA, Douglas JM Jr, Malotte CK, et al. Relative efficacy of prevention counseling with rapid and standard HIV testing: a randomized, controlled trial (RESPECT-2). Sex Transm Dis. 2005;32(2):130-8.

38. Lyss SB, Branson BM, Kroc KA, Couture EF, Newman DR, Weinstein RA. Detecting unsuspected HIV infection with a rapid whole-blood HIV test in an urban emergency department. J Acquir Immune Defic Syndr. 2007;44(4):435-42.

39. Rapid HIV testing in outreach and other community settings-United States, 2004-2006. MMWR Morb Mortal Wkly Rep. 2007;56(47):12337 .

40. Kassler WJ, Dillon BA, Haley C, Jones WK, Goldman A. On-site, rapid HIV testing with same-day results and counseling. Aids. 1997;11 (8): 1045-51.

41. Kroc K, Kendrick S, Withum D et al. Rapid HIV Testing in an Emergency Department. Vol. 2008; 2002.

42. Haukoos JS, Hopkins E, Byyny RL. Patient acceptance of rapid HIV testing practices in an urban emergency department: assessment of the 2006 CDC recommendations for HIV screening in health care settings. Ann Emerg Med. 2008;51(3):303-9. 309 e1; discussion 310-1.

43. Silva A, Glick NR, Lyss SB, et al. Implementing an HIV and sexually transmitted disease screening program in an emergency department. Ann Emerg Med. 2007;49(5):564-72.

44. Farnham PG, Hutchinson AB, Sansom SL, Branson BM. Comparing the costs of HIV screening strategies and technologies in health-care settings. Public Health Rep. 2008;123(Suppl 3):51-62.

45. Mehta SD, Hall J, Greenwald JL, Cranston K, Skolnik PR. Patient risks, outcomes, and costs of voluntary HIV testing at five testing sites within a medical center. Public Health Rep. 2008;123(5):608-17.

46. Walensky RP, Arbelaez C, Reichmann WM, et al. Revising expectations from rapid HIV tests in the emergency department. Ann Intern Med. 2008; 149(3): 153-60.

47. Walensky RP, Arbelaez CA, Reichmann WM, Walls RM, Katz JN, Losina E. Revisiting Expectations from Rapid HIV Test and Confirmation in the Emergency Department [Abstract]. 15th Conference on Retroviruses and Opportunistic Infections. Boston, MA; 2008.

48. Greenwald JL, Burstein GR, Pincus J, Branson B. A rapid review of rapid HIV antibody tests. Curr Infect Dis Rep. 2006;8(2):125-31.

49. Yeni PG, Hammer SM, Hirsch MS, et al. Treatment for adult HIV infection: 2004 recommendations of the International AIDS Society-USA Panel. Jama. 2004;292(2):251-65.

50. Panel on Antiretroviral Guidelines for Adult and Adolescents. Guidelines for the use of antiretroviral agents in HIV-1-infected adults and adolescents. Vol. 2008: Department of Health and Human Services; 2008. 